\section{RECIS}

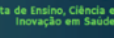

ISSN: $2675-9683$

\section{Revista de Ensino, Ciência e Inovação}

Homepage:http://recis.huunivasf.ebserh.gov.br

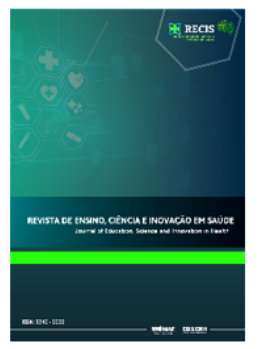

\title{
O exame neurológico em telemedicina: Uma revisão
}

\section{The neurological examination in telemedicine: A review}

\section{Pedro Igor Lustosa Roriz ${ }^{1,2}$, Lara Eline de Souza Menezes ${ }^{2}$, Silvanildo Macário dos Santos Filho $^{3}$, Mariana Barbosa de Araújo ${ }^{3}$}

${ }^{1}$ Neurologista do Departamento de Neurologia do Hospital Memorial Petrolina, ${ }^{2}$ Docente do curso de Medicina da Faculdade Estácio de Sá Juazeiro-BA. ${ }^{3}$ Discente do curso de Medicina da Universidade Federal do Vale do São Francisco - UNIVASF

E-mail para contato: pedroilroriz@gmail.com

Artigo recebido em 20/12/2020 e aceito em 12/04/2021

\begin{abstract}
RESUMO
A fim de manter a continuidade do atendimento ambulatorial diante do curso da pandemia, muitas consultas médicas estão sendo realizadas com o uso de telemedicina. Para pacientes ambulatoriais de neurologia, ainda observamos poucos dados de pesquisa sobre o uso remoto de consultas e apenas evidências de baixo nível para seu uso na prática. Além disso, observamos uma grande escassez, na literatura mundial, de publicações sobre o exame físico neurológico a distância. Diante desse novo contexto de saúde mundial, as próximas gerações de médicos deverão ser formadas para saberem oferecer cuidados de saúde de alta qualidade, de forma segura e individualizada, por meio de teleconsultas. Nesse trabalho realizamos uma revisão de literatura para investigar as práticas que vêm sendo adotadas para realização do exame neurológico em telemedicina, as dificuldades e as soluções encontradas.

Palavras-chave: Exame Neurológico; Telemedicina; Consulta Remota; Neurologia; Teleneurologia
\end{abstract}

\begin{abstract}
:
In order to maintain the consistency of the outpatient care, in the face of the course of the pandemic state, many of the medical consults are being realised with the support of the telemedicine. For outpatients of the neurology, we observed few research data about the remote use of consults, and only low-level evidences for its use in practice. In addition to this, we observed a big scarcity in the world literature, of publications about the neurological physical examination done at a distance. In front of this new world health context, the next generations of doctors should be formed and trained to know how to offer health care of high quality, in a secure and individualized way, through the teleconsultations. In this article, we performed a literature review to investigate the practices that have been adopted to perform the neurological examination in telemedicine, the difficulties and the solutions found.
\end{abstract}

Keywords: Neurologic Examination; Telemedicine; Remote Consultation; Neurology; Teleneurology 


\section{INTRODUÇÃO}

A pandemia de COVID-19 causou uma diminuição importante da disponibilidade de serviços de saúde para pacientes internados e ambulatoriais. A fim de manter a continuidade do atendimento ambulatorial diante do curso da pandemia, por causa das medidas rígidas de distanciamento social, muitas consultas médicas estão sendo realizadas com o uso de telemedicina. ${ }^{1}$

A telemedicina vem sendo estimulada para garantir a continuação dos cuidados para os pacientes, inclusive para aqueles que estão em situação de vulnerabilidade social. ${ }^{2}$

Teleconsultas e telesaúde já vêm sendo amplamente utilizadas no mundo todo, mas não encontrávamos muitos estudos, na literatura mundial, sobre este tema até março de 2020. Essa temática parecia existir de forma quase que imperceptível dentro das escolas de formação em saúde.

A pandemia ofereceu às escolas de saúde uma chance de incorporar o treinamento em telemedicina aos currículos de maneira prática. ${ }^{3} \mathrm{De}$ agora em diante, as próximas gerações de médicos deverão ser formadas para saberem oferecer cuidados em saúde com alta qualidade e de forma segura e individualizada por meio de teleconsultas. ${ }^{3}$

Além das vantagens relacionadas à segurança biológica, $\mathrm{o}$ atendimento remoto diminui os custos para os pacientes e para os serviços de saúde, oferece atendimento especializado para uma população mais abrangente e encurta as distâncias entre o médico e o paciente. ${ }^{2}$

Esse trabalho tem como objetivo identificar estratégias que vem sendo adotadas para realização do exame neurológico em telemedicina, as dificuldades e as soluções encontradas.

\section{MATERIAL E MÉTODOS}

Foi realizado uma revisão sistemática na literatura para identificar os artigos acerca do assunto. Realizou-se busca nas bases Medline e LILACS, de artigos que foram publicados de 2015 até setembro de 2020, usando os seguintes descritores: exame neurológico e telemedicina ou exame neurológico e consulta remota ou teleneurologia. Foram utilizados apenas termos em inglês e buscas manuais foram feitas nas referências bibliográficas dos artigos encontrados.

\section{RESULTADOS E DISCUSSÃO}

Todas as especialidades médicas mergulharam nesse novo contexto de saúde mundial e tentaram se adaptar a essa nova realidade. Entre as especialidades, a neurologia é uma área que sofre para transpor essa dificuldade causada pelo afastamento entre o médico e o paciente.

A parte mais crítica desse impasse, que diminui o interesse dos neurologistas para o atendimento remoto, é a dificuldade para realizar o exame neurológico.

Procedimento essencial para o diagnóstico topográfico e nosológico de um agravo e fundamental para avaliar a necessidade de exames complementares, diminuindo os custos da assistência, é inerente a esse exame o contato físico. Esse contato é exemplificado na avaliação da força, dos reflexos e da sensibilidade.

Entretanto, na atual conjuntura mundial, o interesse nessa modalidade de consulta a distância é muito crescente.

A teleneurologia já tem sido usada há muito tempo, principalmente nos serviços de referência para Acidente Vascular Cerebral (AVC). ${ }^{4,5}$ Já é comprovado que os cuidados do teleAVC chegam até a encurtar a duração da internação hospitalar. ${ }^{4}$ Mas, para os pacientes ambulatoriais de neurologia, ainda observamos poucos dados de pesquisa sobre o uso remoto de consultas e apenas evidências de baixo nível para o seu uso na prática. ${ }^{5}$

Foi encontrado nove artigos publicados, no período pesquisado, que trazem orientações e sugestões para realização do exame físico neurológico em teleconsultas. Todos os trabalhos são baseados na opinião de especialistas e de consensos.

Para o manejo longitudinal de condições crônicas como esclerose múltipla, doença de Parkinson e síndromes demenciais, as visitas de acompanhamento de telemedicina podem funcionar de forma excelente e podem garantir uma boa qualidade de vida para os pacientes. ${ }^{6}$

Embora exista uma grande escassez na literatura mundial, de publicações sobre o exame físico neurológico em telemedicina, ${ }^{7}$ alguns autores começaram a pensar e a produzir até mesmo kits de autoexame, que podem ajudar o neurologista e o paciente nesse processo, mesmo que apenas num formato de esboço experimental. ${ }^{8}$

De forma mais prática, alguns especialistas revelam que, para uma avaliação mais precisa do paciente, devemos seguir alguns princípios: (1) A forma adequada de usar o aparelho eletrônico pelo paciente; (2) A capacidade de reconhecer o normal versus o patológico, pelo médico; (3) A orientação adequada do paciente pelo médico. ${ }^{7}$ 


\section{ORIENTAÇÕES PARA AS CONSULTAS POR VIDEOCONFERENNCIA}

Em relação aos profissionais, a literatura oferece algumas orientações para o adequado controle e organização da consulta. Primeiramente, no que diz respeito aos aplicativos que podem ser usados para essa finalidade, o médico deve utilizar, de preferência, aplicativos específicos e que possuem criptografia de ponta a ponta.

Também é possível utilizar aplicativos de uso pessoal, como o FaceTime, o Google Hangouts, o Whatsapp e o iMessage, já que esses também são criptografados, na tentativa de facilitar o entendimento e o uso da ferramenta pelo paciente. ${ }^{7}$ Lembrando-se de respeitar todos os princípios éticos no compartilhamento de dados e de informações.

Durante a videoconferência, o médico deve usar, preferencialmente, fones de ouvido para obter com mais precisão as informações que estão sendo ditas e para manter a confidencialidade. ${ }^{5} \mathrm{O}$ profissional deve realizar o atendimento em um consultório silencioso e deve manter uma postura calma e receptiva. Antes de iniciar a consulta, deve ser mostrado ao paciente um documento original com foto e o seu registro no conselho de medicina.

As orientações aos pacientes devem ser dadas bem antes da consulta para que eles possam escolher o melhor cômodo da casa e qual a melhor vestimenta para a ocasião. Resumimos as orientações para os pacientes no Quadro 1.

Quadro 1 - Orientações para os pacientes antes da consulta de telemedicina

\begin{tabular}{|l|}
\hline - Certifique-se do horário exato da consulta e \\
evite atrasos; \\
\hline - Escolha um ambiente claro, silencioso, livre de \\
distrações e com espaço para você ficar em pé e \\
andar uma distância curta; \\
\hline - Esteja com seu computador ou smartphone \\
carregado ou ligado na tomada; \\
\hline - Em caso de smartphone, certifique-se de que \\
existe um apoio para ele, pois você deverá estar \\
com as duas mãos livres; \\
\hline - Use roupas confortáveis que não alterem sua \\
mobilidade, de preferência short e camiseta, para \\
facilitar o exame, caso seja necessário; \\
\hline - Durante algumas partes do exame, você deverá \\
ficar a aproximadamente 2 metros da câmera, \\
quando solicitado;
\end{tabular}

Fonte: Adaptado de Wahezi et al. (2020)

Em alguns casos específicos, pode ser solicitado o apoio de cuidadores ou de parentes próximos, como no caso de pacientes que têm problemas de comunicação ou dificuldades cognitivas. ${ }^{5}$

$\begin{array}{lll}O & \text { EXAME } & \text { NEUROLÓGICO } \\ \text { TELEMEDICINA }\end{array}$

Sabe-se que os dados que a história clínica fornece, na grande maioria das vezes, são o que norteiam um diagnóstico. ${ }^{5}$ Dessa forma, é fundamental uma anamnese ainda mais detalhada para suprir as lacunas do exame físico.

Para facilitar ainda mais o entendimento do paciente, durante o exame, devemos realizar os movimentos específicos e pedir para o paciente imitá-los. No quadro 2, observamos o roteiro dos principais itens que podem ser avaliados e algumas dicas para sua realização.

Quadro 2 - Dicas para realização do exame neurológico em telemedicina

\begin{tabular}{|l|l|}
\hline Estado & $\begin{array}{l}\text { Observe se o paciente está } \\
\text { alerta e orientado quanto à } \\
\text { pessoa, o lugar e à hora. Avalie } \\
\text { sua aparência geral, o } \\
\text { autocuidado, o humor e o } \\
\text { comportamento. } \\
\text { - Observe a função da } \\
\text { linguagem durante a anamnese. }\end{array}$ \\
\hline Cranianos & $\begin{array}{l}\text { - Fala / voz. Anote as alterações } \\
\text { percebidas durante a história } \\
\text { clínica. }\end{array}$ \\
- Acuidade visual. Peça para o \\
paciente ler um jornal, por \\
exemplo (com um olho de cada \\
vez). \\
- Movimentos oculares. Peça \\
para o paciente, com a cabeça \\
parada, olhar para a esquerda e \\
para a direita, e depois para \\
cima e para baixo. \\
- Avalie a presença de nistagmo \\
e de simetria pupilar (com o \\
paciente próximo à câmera). \\
- Procure assimetrias faciais \\
(fechar os olhos com força, \\
sorrir e levantar as \\
sobrancelhas). \\
- Peça para mover a mandíbula \\
de um lado para o outro. \\
- Colocar a língua fora da boca \\
(analisar simetria, desvio e \\
atrofia). \\
\hline
\end{tabular}




\begin{tabular}{|c|c|}
\hline $\begin{array}{l}\text { Função } \\
\text { Motora }\end{array}$ & $\begin{array}{l}\text { Trofismo. Tente avaliar } \\
\text { visualmente (paciente com } \\
\text { vestes adequadas). } \\
\text { - Força dos membros } \\
\text { superiores. Realizar prova dos } \\
\text { braços estendidos. Verificar se } \\
\text { há pronação rápida ou } \\
\text { assimetrias. Utilizar elevação } \\
\text { de objetos do dia a dia (p. ex. } \\
\text { garrafa de água) para verificar } \\
\text { fraqueza segmentar. } \\
\text { - Força de membros inferiores. } \\
\text { Pedir ao paciente para se } \\
\text { levantar de uma cadeira. Ficar } \\
\text { na ponta dos pés / calcanhares. }\end{array}$ \\
\hline Coordenação & $\begin{array}{l}\text { - Teste dedo-nariz (paciente } \\
\text { estende o braço totalmente, em } \\
\text { seguida, leva a ponta do dedo } \\
\text { para tocar o nariz). } \\
\text { - Movimentos rápidos } \\
\text { alternados das mãos e dos } \\
\text { dedos. } \\
\text { - Teste de Romberg. }\end{array}$ \\
\hline Marcha & $\begin{array}{lrr}\text { - Peça } & \text { para atravessar o } \\
\text { cômodo. } & \text { Procure alguma } \\
\text { marcha } & \text { característica } \\
\text { (cerebelar, } & \text { parkinsoniana, } \\
\text { ceifante etc.). } & \\
\end{array}$ \\
\hline Outros sinais & $\begin{array}{l}\text { - Descreva a presença de } \\
\text { movimentos anormais e outras } \\
\text { alterações (bradicinesia, coreia, } \\
\text { tremores etc.). }\end{array}$ \\
\hline
\end{tabular}

Fonte: Adaptado de Nitkunan et al. (2020)

Se possível, devemos aprofundar o exame neurológico na queixa específica do paciente e deter mais tempo na parte do exame que julgar necessário. Por exemplo, se o paciente apresentar tremores, uma avaliação mais minuciosa deste movimento anormal deve ser pesquisada (presença de assimetria, sua relação com o repouso e com o movimento, por exemplo).

$\mathrm{Na}$ avaliação da parte motora, podemos utilizar instrumentos do dia a dia (garrafas de água ou de leite), que são encontrados nas casas dos pacientes, transformando o objeto em um instrumento que sirva para verificar a força de forma mais ou menos objetiva, ${ }^{7}$ verificando se um braço tem mais dificuldade para levantar o mesmo objeto que o outro, por exemplo.

$\mathrm{Na}$ parte inicial da avaliação (estado mental e pares cranianos), o paciente deve ficar mais próximo da câmera e do microfone para o examinador perceber, de forma mais clara, as alterações faciais e a voz. Na segunda parte do exame, podemos pedir para o paciente ficar a 2 metros da câmera, distância a partir da qual é possível avaliar melhor as alterações motoras da maior parte do corpo. ${ }^{7}$

O exame e as consultas devem ser adaptados diante do contexto social e da disponibilidade de tecnologia local.

\section{CONCLUSÕES}

Embora os atendimentos por telemedicina tenham aumentando de forma significativa nos últimos tempos, catalisados pela pandemia da COVID-19, e sua necessidade tenha sido comprovada, a nossa literatura ainda carece de mais trabalhos sobre esse tema, principalmente no que tange à prática e à avaliação do exame físico a distância. Esperamos que as instituições de ensino comecem a introduzir essa problemática nas matrizes curriculares e fomentem pesquisas sobre o tema.

\section{REFERÊNCIAS}

1. Sosnowski R, Kamecki H, Joniau S, et al. Introduction of Telemedicine During the COVID-19 Pandemic: A Challenge for Now, an Opportunity for the Future. Eur Urol 2020; S0302-2838(20)30539-X. https://doi.org/10.1016/j.eururo.2020.07.007

2. Bloem BR, Dorsey ER, Okun MS. The Coronavirus Disease 2019 Crisis as Catalyst for Telemedicine for Chronic Neurological Disorders. JAMA Neurol 2020; 75(4):10711072.

https://doi.org/10.1001/jamaneurol.2020.1452

3. Jumreornvong O, Yang E, Race J, Appel $\mathrm{J}$. Telemedicine and Medical Education in the Age of COVID-19. Acad Med 2020;10.1097 . https://dx.doi.org/10.1097\%2FACM.0000000 000003711

4. Blue R, Yang A I, Zhou C, et al. Telemedicine in the Era of Coronavirus Disease 2019 (COVID-19): A Neurosurgical Perspective. World Neurosurg 2020; 139:549-557. https://dx.doi.org/10.1016\%2Fj.wneu.2020.05 .066

5. Nitkunan A, Paviour D, Nitkunan T. COVID19: switching to remote neurology outpatient consultations. Pract Neurol 2020; 20:222-224 http://dx.doi.org/10.1136/practneurol-2020002571

6. Perez DL, Biffi A, Camprodon JA et al. Telemedicine in Behavioral NeurologyNeuropsychiatry: Opportunities and Challenges Catalyzed by COVID-19. Cogn Behav Neurol 2020; 33(3):226- 
229. https://doi.org/10.1097/wnn.000000000 0000239

7. Wahezi SE, Duarte RV, Yerra S, et al. Telemedicine During COVID-19 and Beyond: A Practical Guide and Best Practices Multidisciplinary Approach for the Orthopedic and Neurologic Pain Physical Examination. Pain Physician 2020; 23(Sppl 4):S205-S238.

8. Goyal DKC, Divi SN, Schroeder GD, et al. Development of a Telemedicine
Neurological Examination for Spine Surgery: A Pilot Trial. Clin Spine Surg 2020; 33(9): 355-

369. https://doi.org/10.1097/bsd.00000000000 01066

9. Awadallah M, Janssen F, Körber, B, et al. Telemedicine in General Neurology: Interrater Reliability of Clinical Neurological Examination Via Audio-Visual Telemedicine. Eur Neurol 2018; 80 (5-6): 289294. https://doi.org/10.1159/000497157. 